\title{
Variabilidade espacial e temporal da seção transversal de escoamento superficial em sulcos de irrigação'
}

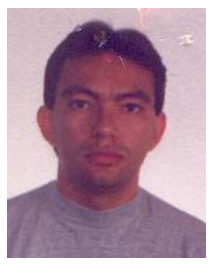

Joaquim J. de Carvalho ${ }^{2}$, Carlos A. V. de Azevedo ${ }^{3}$, Jaqueline Henrique ${ }^{2}$, José Dantas Neto ${ }^{3}$, Vera L. A. de Lima ${ }^{3}$ \& Roberto V. Pordeus ${ }^{4}$

\footnotetext{
1 Parte da dissertação de Mestrado do primeiro autor, apresentada a UFPB, convênio UFPB-Embrapa Algodão-Escola Agrotécnica Federal de Sousa, PB

2 R. Ana Azevedo 509, Palmeira, CEP 58102-320, Campina Grande, PB. Fone: (83) 321-7654 (Foto)

3 UFCG/CCT/DEAg. Av. Aprígio Veloso 882, Bodocongó, CEP 58109-970, Campina Grande, PB. Fone: (83) 310-1361, Fax: (83) 310-1185. E-mail:cazevedo@deag.ufpb.br

${ }^{4}$ Doutorando em Recursos Naturais, UFCG/CCT/DEAg
}

Protocolo 164 - 20/11/2001 - Aprovado em 14/2/2003

\begin{abstract}
Resumo: Apesar do método de irrigação por superfície ser o mais antigo e mais usado no mundo, em virtude de suas vantagens em comparação ao método pressurizado, este tem apresentado baixos índices de desempenho devido ao seu manejo inadequado como conseqüência de dificuldades no monitoramento das variações espacial e temporal nos parâmetros de campo. Assim sendo, esta pesquisa teve o objetivo de estimar a variabilidade espacial e temporal da seção transversal de escoamento superficial em sulcos de irrigação, medindo-se seu perfil após cada evento de irrigação, ao longo da estação de cultivo (total de 12 eventos) em seis sulcos eqüidistantes, de forma a considerar as variações nesse parâmetro, no sentido transversal da área irrigada. As variações máximas observadas, ao longo da estação de cultivo, na largura de topo, na largura a $50 \%$ da altura máxima, na largura da base e na altura máxima foram, respectivamente, em termos espaciais iguais a 16,3,18,7, 105,2 e 35,1\%; e iguais a $11,4,13,5,38,0$ e $23,7 \%$, em termos temporais.
\end{abstract}

Palavras-chave: irrigação por sulco, seção transversal, variabilidade

\section{Spatial and temporal variability of flow cross-sectional area of furrow irrigation}

\begin{abstract}
In spite of surface irrigation method being the oldest and most used in the world due to its advantages compared to pressurized method, it has presented low levels of performance, because its inadequate managemet as a consequence of the difficulties in monitoring the spatial and temporal variabilities of field parameters. Thus, this research had the purpose of estimating the spatial and temporal variability of the flow cross-sectional area of irrigation furrows, measuring its profile after each irrigation event, along the crop season (total of 12 events) in six furrows evenly spaced in a way to comprise the variations in this parameter along the cross-sectional irrigated area. The maximum variations observed, along the crop season, in the topwidth, width at $50 \%$ of the maximum height, base width and maximum height were, respectively, in spatial terms equal to $16.3,18.7,105.2$ and $35.1 \%$; and equal to $11.4,13.5,38.0$ and $23.7 \%$, in temporal terms.
\end{abstract}

Key words: furrow irrigation, cross-sectional area, variability

\section{INTRODUÇÃO}

Segundo Daker (1988) a forma geométrica da seção transversal dos sulcos, incluindo sua profundidade, é muito importante na eficiência e adequabilidade da irrigação; as dimensões dessa seção devem, sempre que possível, basearse no tipo de solo e da cultura e na declividade do sulco. Para solos com baixa capacidade de infiltração e sulcos de pequena declividade, a seção deve ser maior na largura que na profun- didade. Em sulcos com maiores declividades, a sua seção deve ser, de preferência, em forma de $\mathrm{V}$.

O conhecimento dos parâmetros geométricos da seção transversal do sulco torna-se necessário para a caracterização do escoamento superficial, através de equações de escoamento superficial. A área da seção transversal é requerida para se calcular o armazenamento superficial d'água e a velocidade de escoamento superficial, a profundidade para se determinar a elevação da água e, assim, o declive da superfície de fricção, 
utilizados nos modelos de simulação da irrigação superficial (zero-inércia, hidrodinâmico, ondas cinemáticas e balanço de volume); além do mais, a infiltração acumulada tem relação com o perímetro molhado (Trout,1991).

Para propósitos de avaliação e manejo da irrigação, devese medir a seção transversal de cada sulco selecionado, pelo menos em duas ou três estações (Walker \& Skogerboe, 1987) havendo variedade de técnicas que podem ser utilizadas para essa finalidade. Walker \& Cahoon (1989) apud Cahoon (1995) descrevem perfilômetros com barras corrediças que podem ser usados para medir a profundidade do sulco em diferentes posições. De acordo com Machado et al. (1996) a constituição, a precisão e o espaçamento entre as hastes do perfilômetro, podem variar de equipamento para equipamento, sendo que uma das maiores limitações desse tipo de equipamento é a tomada de dados no campo, trabalhosa e demorada. Walker (1989) apud Carvalho et al. (1995) utilizando nove perfis de sulcos (três triangulares, três parabólicos e três trapezoidais), desenvolveu uma metodologia para caracterizar a forma geométrica do sulco através de uma análise visual no campo.

De acordo com Carvalho et al. (1995) o advento do uso de modelos matemáticos computacionais tem possibilitado a introdução do perfil geométrico dos sulcos em simulações que visam ao dimensionamento e avaliação do sistema de irrigação. Descrições matemáticas empíricas da seção transversal de sulcos são usadas nas formulações de modelos, muitas vezes como meio de se reduzir a quantidade de informações a serem fornecidas pelos usuários a esses modelos, necessárias à obtenção da solução (Cahoon, 1995). Os modelos SIRTOM (Azevedo, 1992; Azevedo et al. 1996, Azevedo et al., 1997a,b), SIRMOD (modelo desenvolvido por Dr. Wynn R. Walker, Utah State University, USA) e do balanço de volume de Elliott \& Walker (1980) permitem ao usuário fornecer apenas a forma da geometria do sulco e aquelas dimensões básicas de sua seção, como as larguras a $100 \%$ e a $50 \%$ da altura de topo, a base e a altura de topo. Com tais informações, esses modelos ajustam equações do tipo potencial para descreverem a seção transversal e o perímetro molhado em função da altura de escoamento superficial, que é um parâmetro mais facilmente mensurável em campo. Outra opção é permitir ao usuário introduzir parâmetros geométricos empíricos, externamente calculados, para se definir a seção transversal. O modelo SRFR (Strelkoff, 1993 apud Cahoon, 1995) pode ser usado para definir a simetria trapezoidal, ou para entrar com os valores de parâmetros que estabelecem a relação entre profundidade de escoamento superficial e largura de topo.

De acordo com Olitta (1977) o primeiro evento de irrigação modifica sensivelmente a seção transversal do sulco, tendendo a ser parabólica em solos de textura média e triangular ou trapezoidal, em solos de textura fina. Conforme Brown et al. (1988) apud Andrade et al. (1998) a forma do sulco se modifica devido a ação, principalmente da água, a qual quebra os pequenos torrões, carregando-os e depositando-os em novo local; os agregados do solo seco se desfazem à jusante e são rapidamente carreados pelo avanço da água no sulco, devido a força de cisalhamento e pelo próprio escoamento superficial da água; os pequenos aglomerados resultantes, juntamente com as partículas soltas de solo, rolam ao longo do fundo do sulco, enchendo as rachaduras ou cavidades existentes em seu perímetro.

Henrique (1996) e Henrique et al. (1997) monitorando ao longo do tempo e do espaço as características geométricas da seção transversal de sulcos de um sistema de irrigação do Perímetro Irrigado de São Gonçalo, PB, num solo de textura franco-arenosa, observaram variações espaciais e temporais nas dimensões básicas da seção transversal ocorrendo, em geral, aumento nas larguras da base, do topo e a $50 \%$ da altura de topo acompanhado, no entanto, de diminuição nessa altura; devido a essas variações, a geometria do sulco tendeu a uma forma parabólica. Em estudo similar no mesmo perímetro irrigado mas num solo de classificação textural franco, Santana (1996) e Santana et al. (1997) também identificaram variações tanto espaciais como temporais na seção transversal de escoamento superficial dos sulcos, cuja base foi a dimensão, que se mostrou mais vulnerável a alterações ao longo da estação de cultivo, com uma variação de $63 \%$.

O objetivo deste estudo foi avaliar a variabilidade espacial e temporal da seção transversal de escoamento superficial em sulcos, num solo franco-arenoso.

\section{MATERIAL E MÉTODOS}

O trabalho foi desenvolvido num sistema de irrigação por sulcos, da estação experimental da Embrapa Algodão, localizada no perímetro irrigado de São Gonçalo, Sousa, PB. A área experimental cultivada com algodão, apresenta classe textural do tipo franco-arenosa (análise granulométrica (\%), respectivamente nas profundidades de $0-0,30$ e $0,30-0,60 \mathrm{~m}$ : areia $-57,55$ e 62,93; silte $-29,28$ e 22,90; argila-12,49 e 9,23), com uma área de 0,62 ha, foi sistematizada com declividade de $0,004 \mathrm{~m} \mathrm{~m}^{-1}$ no sentido longitudinal e $0,001 \mathrm{~m} \mathrm{~m}^{-1}$ no sentido transversal. O sistema de irrigação possui 53 sulcos com $73 \mathrm{~m}$ de comprimento espaçados $1,6 \mathrm{~m}$.

Para se observar as variações espacial e temporal na seção transversal de escoamento superficial dos sulcos, realizaramse medições de sua perfilometria para cada evento de irrigação ao longo da estação de cultivo (total de 12 eventos) em seis sulcos eqüidistantes, de forma a abranger ao máximo essas variações, ao longo de toda a área irrigada. Em cada um desses sulcos a perfilometria foi medida em três estações, eqüidistantes $18,25 \mathrm{~m}$ ao longo do sulco, utilizando-se um perfilômetro (Figura 1) confeccionado pelo Laboratório de Engenharia de Irrigação e Drenagem do Departamento de Engenharia Agrícola da UFCG. A tomada desses dados foi feita apenas quando a fase de recessão se completou; no entanto, durante a fase de armazenamento, quando o perfil superficial de escoamento se tornou praticamente estável, foram colocadas pequenas estacas nessas estações demarcando-se, assim, a seção transversal de escoamento superficial a ser medida posteriormente. As dimensões da seção transversal de escoamento que foram medidas estão ilustradas na Figura 2.

Os dados obtidos experimentalmente foram analisados utilizando-se métodos de estatística descritiva e se calculando a média aritmética, os valores mínimo e máximo, a amplitude 


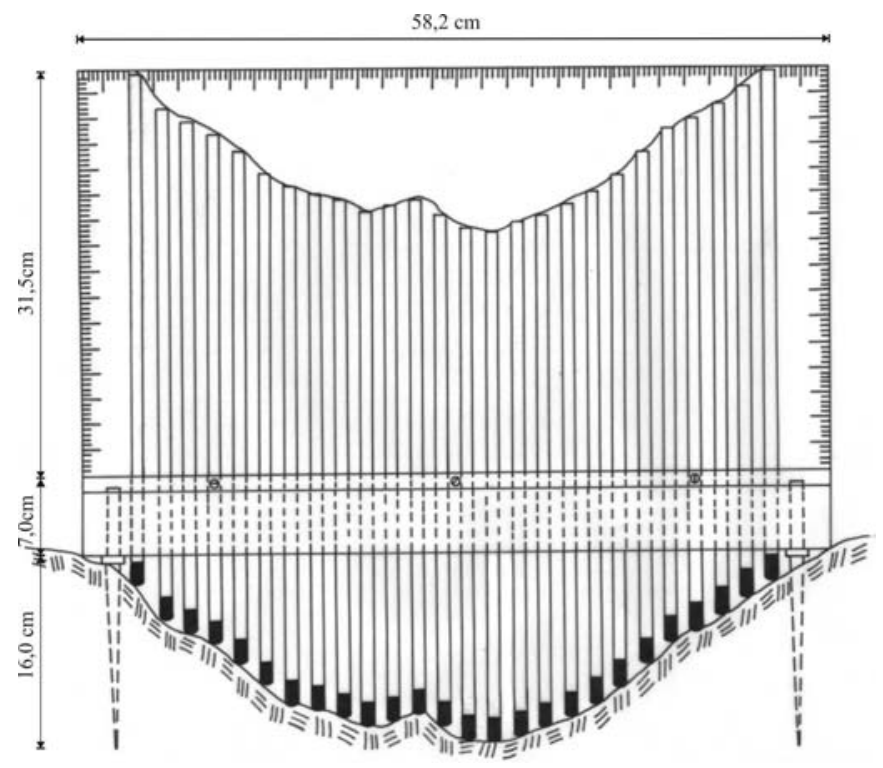

Figura 1. Ilustração do perfilômetro utilizado (escala 1:4)

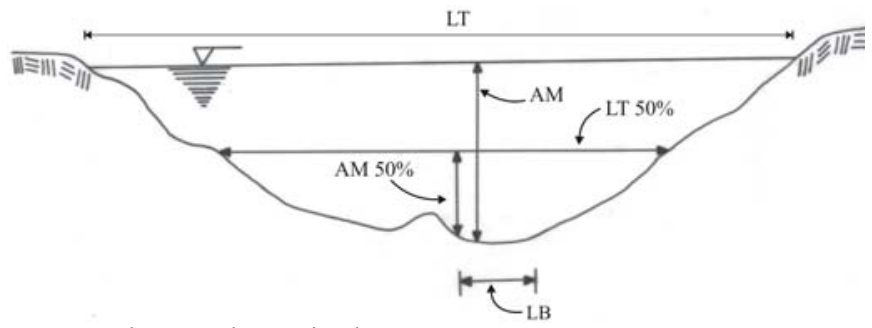

LT - Largura do topo na altura máxima de escoamento

LT $50 \%$ - largura to topo a $50 \%$ de LT

LB - largura da base

AM - altura máxima de escoamento

AM $50 \%-50 \%$ da altura máxima de escoamento

Figura 2. Ilustração das dimensões da seção transversal de escoamento medidas

total, o intervalo de $95 \%$ de confiança (com desvio-padrão populacional desconhecido) e coeficiente de variação. E, para se avaliar a hipótese de normalidade dos dados, utilizou-se o método de Kolmogorov-Smirnov a nível de 5\% de probabilidade, apud Costa Neto (1997).

\section{RESULTADOS E DISCUSSÃO}

A Tabela 1 (A, B, C e D) apresenta, respectivamente, as variações espacial e temporal nas larguras de topo, a $50 \%$ da altura máxima e da base, e na altura máxima da seção transversal de escoamento superficial, para um solo franco-arenoso. Geometricamente, a Figura 3 exibe as variações na seção transversal de escoamento superficial nos sulcos, ocorridas na estação $36,5 \mathrm{~m}$. Os valores das referidas dimensões, específicos a cada sulco estudado, num determinado evento de irrigação, representam médias de medições em três estações ao longo do sulco. Verificou-se, através da análise da distribuição de freqüência dos dados, que os parâmetros geométricos da seção transversal dos sulcos de irrigação apresentaram distribuição normal.

Observa-se na Tabela 1 (A, B, C e D) que, tanto ao longo do tempo quanto no sentido transversal da área irrigada, a desuniformidade foi mais expressiva na largura da base. Verifica-se pelo coeficiente de variação $(\mathrm{CV})$ dos parâmetros geométricos
Tabela 1. Variação espacial e temporal dos parâmetros da seção transversal de escoamento superficial média dos sulcos de irrigação

\begin{tabular}{|c|c|c|c|c|c|c|c|c|}
\hline \multirow{2}{*}{$\begin{array}{l}\text { Eventos de } \\
\text { Irrigação }\end{array}$} & \multicolumn{6}{|c|}{ Sulcos de Irrigação } & \multirow{2}{*}{$\begin{array}{l}\text { Média } \\
\text { Espacial }\end{array}$} & \multirow{2}{*}{$\begin{array}{l}C V^{\mathrm{e}} \\
(\%)\end{array}$} \\
\hline & 1 & 2 & 3 & 4 & 5 & 6 & & \\
\hline \multicolumn{9}{|c|}{ A. Largura de topo, LT (m) } \\
\hline 1 & 0,330 & 0,315 & 0,302 & 0,281 & 0,276 & 0,280 & 0,297 & 7,4 \\
\hline 2 & 0,385 & 0,359 & 0,379 & 0,333 & 0,286 & 0,318 & 0,343 & 11,1 \\
\hline 3 & 0,378 & 0,356 & 0,247 & 0,327 & 0,286 & 0,274 & 0,311 & 16,3 \\
\hline 4 & 0,409 & 0,335 & 0,306 & 0,329 & 0,329 & 0,277 & 0,331 & 13,3 \\
\hline 5 & 0,405 & 0,368 & 0,282 & 0,329 & 0,276 & 0,309 & 0,328 & 15,4 \\
\hline 6 & 0,423 & 0,342 & 0,295 & 0,377 & 0,303 & 0,295 & 0,339 & 15,4 \\
\hline 7 & 0,343 & 0,360 & 0,283 & 0,355 & 0,282 & 0,313 & 0,323 & 10,9 \\
\hline 8 & 0,389 & 0,347 & 0,290 & 0,270 & 0,297 & 0,280 & 0,312 & 14,8 \\
\hline 9 & 0,417 & & 0,332 & 0,275 & 0,310 & 0,315 & & 14,3 \\
\hline 10 & 0,412 & 0,353 & 0,327 & 0,307 & 0,349 & 0,314 & 0,344 & 11,1 \\
\hline 11 & 0,409 & 0,327 & 0,342 & 0,378 & 0,336 & 0 , & 0,356 & 8,7 \\
\hline 12 & 0,38 & 0,332 & 0,322 & 0,3 & 0,282 & & & 11,0 \\
\hline Média & 391 & 0,344 & 309 & 0,327 & 30 & 0.303 & 0.329 & \\
\hline Temporal & & & 09 & & & & & \\
\hline CV $(\%)$ & 7,4 & 4,6 & 11,1 & 11,4 & 8,3 & 7,3 & & 12,7 \\
\hline \multicolumn{9}{|c|}{ B. Largura a $50 \%$ da altura máxima, LT50\% (m) } \\
\hline 1 & 0210 & (1) & 215 & 0,178 & 0,202 & 0,167 & 00 & 11,3 \\
\hline 2 & 0,246 & 19 & 0,228 & 0,2 & & & & 5,2 \\
\hline 3 & 0,242 & 0,233 & 0,162 & 0,232 & 0,216 & 0,197 & 0,214 & 14,0 \\
\hline 4 & 0,245 & 0,241 & 0,185 & 0,237 & 0,189 & 0,196 & 0,216 & 13,1 \\
\hline 5 & 0,246 & 0,209 & 0,179 & 0,222 & 0,207 & 0,228 & 0,215 & 10,5 \\
\hline 6 & 0,274 & 0,246 & 0,205 & 0,257 & 0,212 & 0,158 & 0,225 & 18,7 \\
\hline 7 & 0,186 & 0,193 & 0,194 & 0,232 & 0,144 & 0,193 & 0,190 & 14,7 \\
\hline 8 & 0,244 & 0,243 & 0,200 & 0,198 & 0,191 & 0,148 & 0,204 & 17,6 \\
\hline 9 & 0,279 & 0,214 & 0,200 & 0,195 & 0,218 & 0,197 & 0,217 & 14,6 \\
\hline 10 & 0,257 & 0,211 & 0,212 & 0,187 & 0,223 & 0,200 & 0,215 & 11,1 \\
\hline 11 & 0,249 & 0,189 & 0,204 & 0,242 & 0,242 & 0,2 & 0,226 & 10,7 \\
\hline 12 & 0,214 & 0,175 & 0,1 & 0,228 & 0,196 & 0,199 & 0,200 & 9,4 \\
\hline Média & 0,241 & 0,217 & 0,198 & 0,219 & 0,205 & 0,195 & 0,212 & \\
\hline CV $(\%)$ & 10,9 & 10,5 & 8,9 & 11,1 & 11,8 & 13,5 & & 13,1 \\
\hline
\end{tabular}

C. Largura da base, LB (m)

$\begin{array}{lllllllll}1 & 0,034 & 0,034 & 0,047 & 0,049 & 0,042 & 0,048 & 0,042 & 16,3\end{array}$

$\begin{array}{lllllllllll}2 & 0,021 & 0,017 & 0,022 & 0,125 & 0,028 & 0,026 & 0,040 & 105,2\end{array}$

$\begin{array}{lllllllll}3 & 0,053 & 0,020 & 0,053 & 0,070 & 0,031 & 0,044 & 0,045 & 39,3\end{array}$

$\begin{array}{lllllllll}4 & 0,030 & 0,026 & 0,041 & 0,079 & 0,030 & 0,042 & 0,041 & 47,3\end{array}$

$\begin{array}{llllllllll}5 & 0,072 & 0,035 & 0,049 & 0,052 & 0,065 & 0,042 & 0,053 & 26,5\end{array}$

$\begin{array}{lllllllll}6 & 0,043 & 0,037 & 0,031 & 0,034 & 0,047 & 0,030 & 0,037 & 18,3\end{array}$

$\begin{array}{lllllllll}7 & 0,022 & 0,021 & 0,041 & 0,060 & 0,057 & 0,053 & 0,042 & 41,1\end{array}$

$\begin{array}{lllllllll}8 & 0,036 & 0,023 & 0,059 & 0,049 & 0,074 & 0,065 & 0,051 & 37,2\end{array}$

$\begin{array}{llllllllll}9 & 0,052 & 0,048 & 0,064 & 0,049 & 0,061 & 0,044 & 0,053 & 14,8\end{array}$

$10 \quad \begin{array}{lllllllll}0,032 & 0,021 & 0,079 & 0,060 & 0,037 & 0,036 & 0,044 & 48,2\end{array}$

$\begin{array}{llllllllll}11 & 0,031 & 0,046 & 0,041 & 0,045 & 0,037 & 0,062 & 0,044 & 24,1\end{array}$

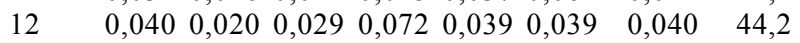

$\begin{array}{lllllllll}\text { Média } & 0,039 & 0,029 & 0,046 & 0,062 & 0,046 & 0,044 & 0,044\end{array}$

$\begin{array}{cccccccc}\text { Temporal } & & & \end{array}$

D. Altura máxima, AM (m)

\begin{tabular}{ccccccccc}
1 & 0,083 & 0,088 & 0,076 & 0,054 & 0,072 & 0,054 & 0,071 & 20,2 \\
2 & 0,099 & 0,089 & 0,072 & 0,043 & 0,059 & 0,065 & 0,071 & 28,6 \\
3 & 0,094 & 0,087 & 0,041 & 0,063 & 0,066 & 0,058 & 0,068 & 28,6 \\
4 & 0,105 & 0,089 & 0,057 & 0,067 & 0,080 & 0,058 & 0,076 & 24,9 \\
5 & 0,082 & 0,079 & 0,052 & 0,068 & 0,057 & 0,061 & 0,067 & 18,2 \\
6 & 0,086 & 0,088 & 0,054 & 0,080 & 0,072 & 0,056 & 0,073 & 20,3 \\
7 & 0,063 & 0,079 & 0,048 & 0,074 & 0,122 & 0,058 & 0,074 & 35,1 \\
8 & 0,084 & 0,091 & 0,048 & 0,057 & 0,061 & 0,046 & 0,065 & 29,1 \\
9 & 0,089 & 0,086 & 0,055 & 0,057 & 0,071 & 0,057 & 0,069 & 22,2 \\
10 & 0,081 & 0,106 & 0,061 & 0,058 & 0,083 & 0,059 & 0,075 & 25,4 \\
11 & 0,081 & 0,051 & 0,076 & 0,089 & 0,075 & 0,071 & 0,074 & 17,3 \\
12 & 0,077 & 0,077 & 0,061 & 0,077 & 0,062 & 0,070 & 0,071 & 10,8 \\
Média & 0,085 & 0,084 & 0,058 & 0,066 & 0,073 & 0,059 & 0,071 & \\
Cmporal & & & 0,07 & & & \\
V (\%) & 12,7 & 15,3 & 19,4 & 19,6 & 23,7 & 11,5 & & 22,8 \\
\hline
\end{tabular}




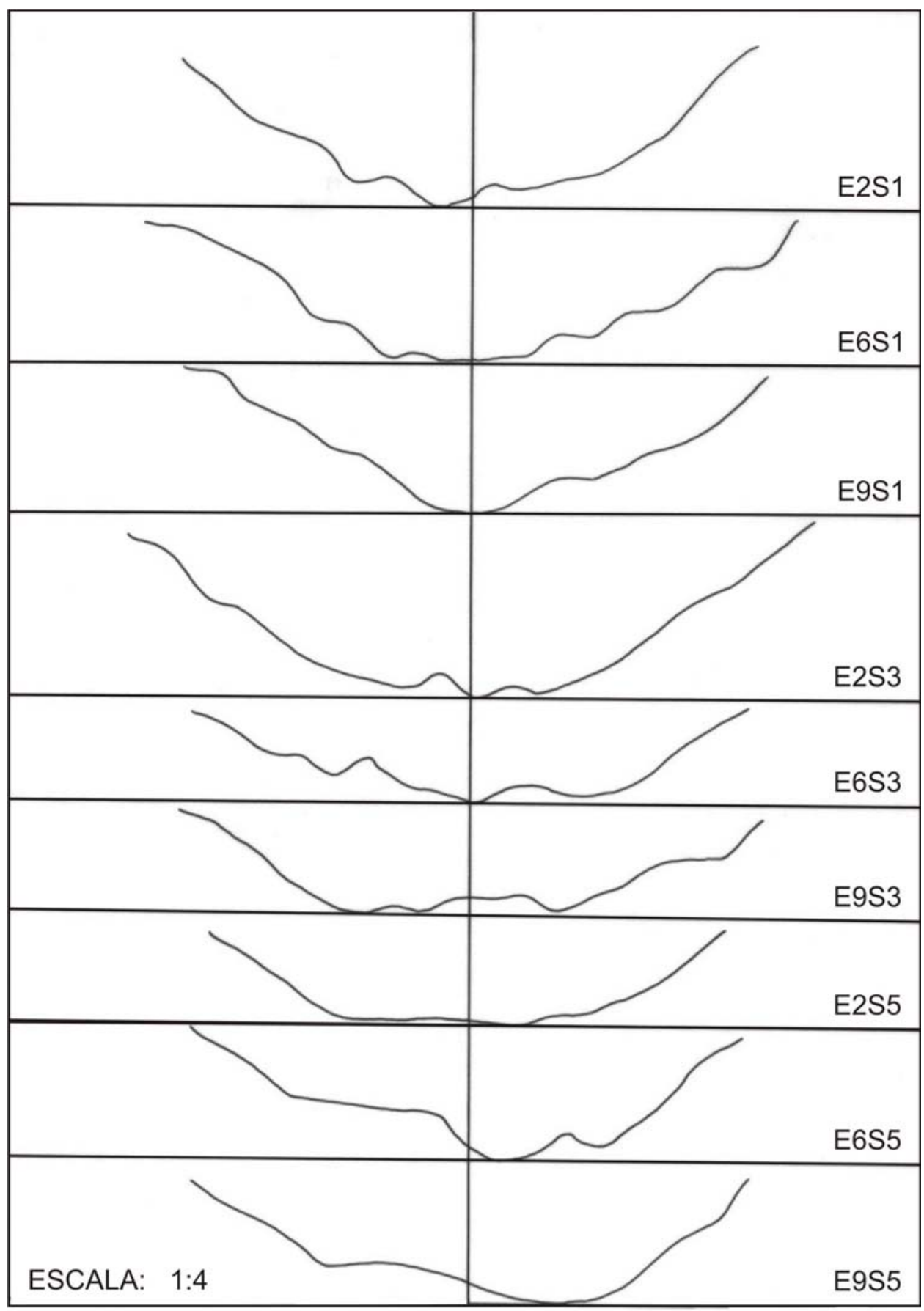

E - Evento de Irrigação; S - Sulco de Irrigação

Figura 3. Variação espacial e temporal da seção transversal de escoamento superficial, referente à estação 36,5 m, nos sulcos de irrigação

largura de topo, largura a $50 \%$ da altura máxima, largura da base e altura máxima da seção transversal dos sulcos que, ao longo da estação de cultivo, as variações espaciais oscilaram de 7,4 a $16,3 \%$, de 5,2 a $18,7 \%$, de 14,8 a $105,2 \%$ e de 10,8 a $35,1 \%$, ao passo que, ao longo do sentido transversal da área irrigada, as variações temporais oscilaram de 4,6 a $11,4 \%$, de 8,9 a $13,5 \%$, de 26,2 a $38,0 \%$, e de 11,5 a $23,7 \%$, respectivamente. Portanto, com base nos limites de CV sugeridos por Warrick \& Nielsen (1980) pode-se dizer que, ao longo do tempo, a largura de topo, a largura a $50 \%$ da altura máxima e a altura máxima apresentaram variações espaciais de baixa $(\mathrm{CV}<12 \%)$ a média
$(12 \% \leq \mathrm{CV} \leq 60 \%)$ e que a largura da base de média a alta $(\mathrm{CV}>$ $60 \%$ ). Ao longo do sentido transversal da área irrigada, a variação temporal apresentada pela largura de topo foi baixa, pela largura a $50 \%$ da altura máxima e a altura máxima foi de baixa a média, e pela largura da base foi média. Nos eventos 3 , 6,2 e 7 e sulcos de irrigação 3,6 , 4 e 5 ocorreram as maiores variações espaciais e temporais na largura de topo, na largura a 50\% da altura máxima, na largura da base e na altura máxima, respectivamente, pois esses eventos e sulcos de irrigação apresentaram maiores coeficientes de variação, estimativas de intervalo a $95 \%$ de confiança e proporções entre a amplitude 
total e a média aritmética, com exceção do parâmetro geométrico largura a $50 \%$ da altura máxima, cujo sulco 5 foi o que apresentou maior proporção entre a amplitude total e a média aritmética, e não o sulco 6, como era esperado, em virtude de apresentar maior coeficiente de variação. Através do procedimento proposto por Hoaglin (1983) verificou-se que isto se deve à ocorrência de um valor discrepante $(0,144 \mathrm{~m}) \mathrm{da}$ largura a $50 \%$ da altura máxima no sulco 5 .

Esta acentuada desuniformidade no parâmetro geométrico largura da base da seção transversal dos sulcos de irrigação, já era esperada visto que a deposição da massa de solo se originaria do desmoronamento do talude do sulco durante as irrigações. Henrique et al. (1997) e Santana et al. (1997), identificaram, em estudos similares em solos franco-arenoso e franco, respectivamente, variações, tanto espaciais como temporais, na seção transversal de escoamento superficial dos sulcos, em que a base do sulco foi a dimensão que se mostrou mais vulnerável a alterações ao longo da estação de cultivo, acompanhada da altura máxima de escoamento superficial, que foi o parâmetro geométrico que apresentou o segundo maior grau de variação, tanto ao longo do tempo quanto espacialmente; o que se deve ao fato da altura máxima de escoamento superficial depender tanto das alterações na largura da seção como das grandezas da vazão e da infiltração d'água; do tempo de aplicação nem tanto, porque suas grandezas permitem, em geral, que o solo atinja a capacidade de infiltração básica, além da vazão de entrada, teoricamente, se manter constante ao longo de sua aplicação.

As médias e os coeficientes de variação apresentados ao longo do tempo e do espaço (Tabela 1), pelos parâmetros geométricos largura de topo, largura a 50\% da altura máxima, largura da base e altura máxima da seção transversal média dos sulcos, foram de $0,329,0,212,0,044$ e $0,071 \mathrm{~m}$, e de $12,7,13,1$, 41,0 e $22,8 \%$, respectivamente. As variações ocorridas sejam ao longo do tempo ou no sentido transversal da área irrigada, que resultaram numa geometria cada vez mais parabólica (Figura 3) são devidas ao desmoronamento do talude dos sulcos em razão do movimento da água, às variações de um evento de irrigação a outro, como entre os sulcos, ocorridas na vazão de entrada e na capacidade de infiltração, e ao surgimento de ervas daninhas dentro dos sulcos.

Os resultados desta pesquisa mostram a necessidade de um monitoramento sistemático, tanto ao longo do tempo como do espaço, das variações nos parâmetros geométricos da seção transversal de escoamento superficial dos sulcos, para se ter uma avaliação do desempenho do sistema de irrigação baseada em características de escoamento superficial e armazenamento superficial d'água nos sulcos, determinados de forma o mais judiciosa possível, capazes, inclusive, de representar ao máximo as condições globais de campo.

Os autores recomendam que pesquisas futuras sejam desenvolvidas no propósito de se avaliar a variabilidade espacial da seção transversal de escoamento superficial no sentido longitudinal dos sulcos; mesmo porque quando se avalia o desempenho desse sistema, as medições dos parâ- metros de campo são feitas apenas em um sulco, supostamente representativo da área.

\section{CONCLUSÕES}

1. As variações espaciais máximas ao longo da estação de cultivo, foram iguais a $16,3,18,7,105,2$ e $35,1 \%$, respectivamente, na largura de topo, na largura a $50 \%$ da altura máxima, na largura da base e na altura máxima.

2. Espacialmente, as variações temporais máximas foram iguais a $11,4,13,5,38,0$ e $23,7 \%$, respectivamente, na largura de topo, na largura a $50 \%$ da altura máxima, na largura da base e na altura máxima.

3. Os resultados desta pesquisa trazem à tona a importância de se conhecer as variações espacial e temporal nos parâmetros geométricos da seção transversal de escoamento superficial dos sulcos, tendo em vista um melhor dimensionamento e manejo desse sistema.

\section{LITERATURA CITADA}

Andrade, D.V. De; Botrel, T.A.; Silva Paz, V.P. Da; Frizzone, J.A. Modelo matemático para simulação da fase de avanço na irrigação por sulcos. Revista Brasileira de Engenharia Agrícola e Ambiental, Campina Grande, v.2 n.1, p.56-62, 1998. Azevedo, C.A.V. de. Real-time solution of the inverse furrow advance problem. Logan: Utah State University, 1992, 263p. Ph.D. Thesis

Azevedo, C.A.V.; Merkley, G.P.; Walker, W.R. The SIRTOM software - a real-time surface irrigation decision support system. International Conference on Computers in Agriculture, 6, Cancun, Mexico, 1996, p.872-884.

Azevedo, C.A.V.; Walker, W.R.; Merkley, G.P. Modelo de ondas cinemáticas na solução do problema inverso da irrigação por sulco em tempo real. Revista Brasileira de Engenharia Agrícola e Ambiental, Campina Grande, v. 1, p. 35-41, 1997a.

Azevedo, C.A.V.; Walker, W.R.; Merkley, G.P. Otimização nãolinear na solução do problema inverso da irrigação por sulcos em tempo real. Revista Brasileira de Engenharia Agrícola e Ambiental, Campina Grande, v.1, p.43-49, 1997b.

Cahoon, J.E. Defining furrow cross section. Journal of Irrigation and Drainage Engineering, ASCE, New York, 1995. v.121, n.1, p.114-119.

Carvalho, D.F. De; Oliveira, L.F.C. De; Pruski, F.F.; Soares, A.A.; Mantovani, E.C. Influência do perfil geométrico do sulco no desempenho da irrigação. In: Congresso Brasileiro de Engenharia Agrícola, 24, 1995, Viçosa. Anais...Viçosa: Sociedade Brasileira de Engenharia Agrícola, 1995. p.222.

Costa Neto, P.L. de O. Estatística. 15 ed. São Paulo: Edgard Blücher, 1997. 264p.

Daker, A. Irrigação e Drenagem; A água na agricultura. 7.ed. Rio de Janeiro: Livraria Freitas Bastos, 1988. v.3. 543p.

Elliott, R.L.; Walker, W.R. Furrow irrigation infiltration and advance functions. Transactions of the ASAE, St. Joseph, 1980. v.25, n.2, p.396-400. 
Henrique, J. Influência dos parâmetros de campo no desempenho dos sistemas de irrigação por sulcos. Campina Grande: Universidade Federal da Paraíba, 1996. 66p. Dissertação Mestrado

Henrique, J.; Azevedo, C.A.V.; Barreto, A.N.; Bezerra, J.R.C.; Dantas Neto, J. Variabilidade espacial e temporal na seção transversal de sulcos no perímetro irrigado de São GonçaloPB. In: Congresso Brasileiro de Engenharia Agrrícola, 26. 1997. Anais... Campina Grande: SBEA, 1997. CD-Rom

Hoaglin, D.C.; Mosteller, F.; Tykey, J.W. Análise exploratória de dados: técnicas robustas, um guia. Lisboa: Editora Salamandra, 1983. 446p.

Machado, M.C.; Oliveira, A.D.; Freitas, M.C.; Vieira, L.B. Influência do número de hastes do perfilômetro na avaliação da rugosidade superficial de um solo. In: Congresso Brasileiro de Engenharia Agrícola, 25. 1996. Bauru: SBEA, 1996. Resumos.

Olitta, A.F.L. Os métodos de irrigação. São Paulo: Nobel; $1977,267 \mathrm{p}$.
Santana, E.F. Monitoramento sazonal do desempenho de um sistema de irrigação por sulco no perímetro irrigado de São Gonçalo, PB. Campina Grande: Universidade Federal, 1996. 96p. Dissertação Mestrado

Santana, E.F.; Azevedo, C.A.V.; Barreto, A.N.; Dantas Neto, J.; Bezerra, J.R.C. Monitoramento sazonal e temporal do perfil geométrico do sulco na cultura do algodoeiro. In: Congresso Brasileiro de Algodão, 1. 1997. Anais... Fortaleza: EMBRAPA, 1997.p.376-379.

Trout, T.J. Furrow geometric parameters. Journal of Irrigation and Drainage Enginneering, ASCE, New York, v. 117, n. 5, p.613-634, 1991.

Walker, W.R.; Skogerboe, G.V. Surface Irrigation: Theory and Practice. 1 ed. Englewood Cliffs: Prentice-Hall, Inc., 1987. 470p.

Warrick, A.W.; Nielsen, D.R. Spatial variability of soil physical properties in the field. In: Hillel, D. (ed.) Application of soil physics. New York: Academic Press, 1980. cap. 13, p.319-344. 